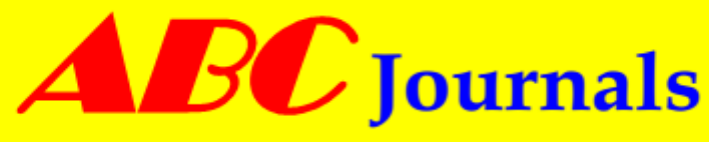

A new domain in research publishing

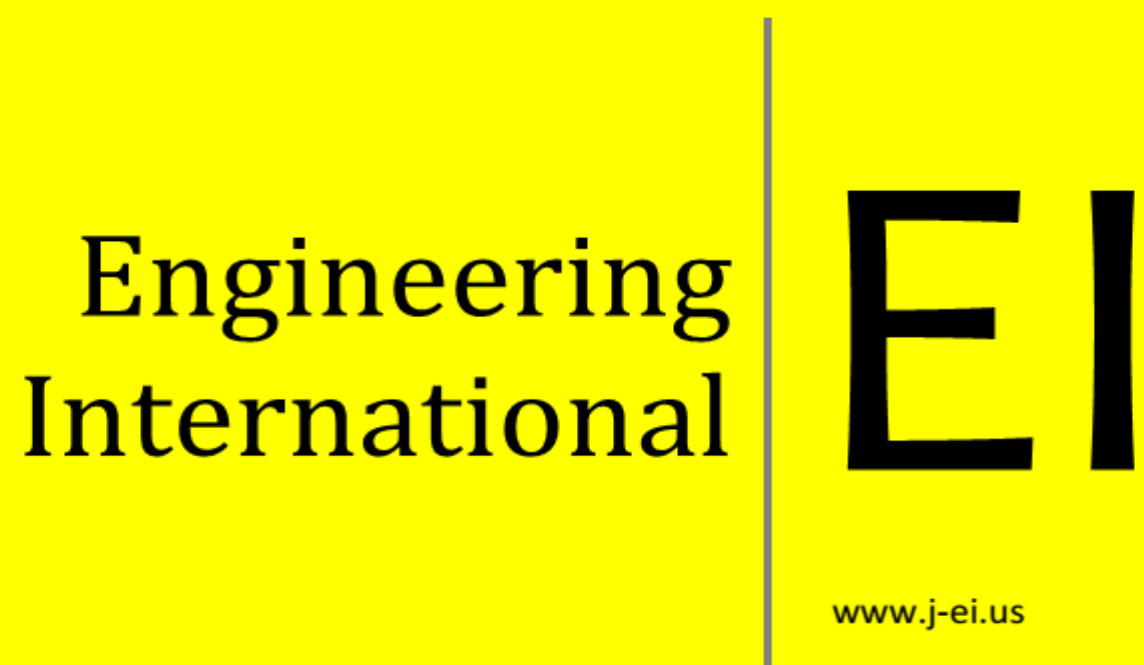

Asian Business Consortium 


\title{
Improved Understanding of $k-\varepsilon$ Turbulence Model for Non-homogeneous Two-Phase Flows in Industrial Combustor
}

\section{Nguyen Thanh Hao}

Industrial University of HoChiMinh City, Vietnam

\begin{abstract}
This paper presents development of $\mathrm{k}-\varepsilon$ turbulence model which can be applied for non-homogeneous two-phase turbulent flows. The improvement of governing equations of $\mathrm{k}-\varepsilon$ turbulence model is based on the decomposition of the two-phase flows into the gas phase and the second phase. Thus, the turbulent kinetic energy $\mathrm{k}$ and the turbulent dissipation rate $\varepsilon$ variables in k- $\varepsilon$ two-equation model are substituted by the turbulent kinetic energy $\mathrm{kg}$ of the gas phase, the turbulent kinetic energy $\mathrm{kp}$ of the second phase, the turbulent dissipation rate $\varepsilon g$ of the gas phase and the turbulent dissipation rate $\varepsilon p$ of the second phase. The new turbulence model is kg-kp$\varepsilon g-\varepsilon p$ four-equation model which can be used to solve all of two-phase flows in nature studies and engineering applications.
\end{abstract}

Keywords: Turbulence model, Non-homogeneous,Two-phase flows, Turbulent kinetic energy, Turbulent dissipation rate;

\section{INTRODUCTION}

History effects are taken into account by the one-equation and two-equation models, where the convection and diffusion of turbulence is modeled by transport equations. The most widely used one-equation turbulence model is due to Spalart and Allmaras (1992), which is based on an eddy-viscosity like variable. The model is numerical very stable and easy to implement on structured as well as unstructured grids. In the case of the two-equation models, practically all approaches employ the transport equation for the turbulent kinetic energy. Among a large number of two-equation models, the k- $\varepsilon$ model of Launder and Spalding and the k- $\omega$ model of Wilcox (1988) are most often used in engineering applications. They offer a reasonable compromise between computational effort and accuracy.

The k- $\varepsilon$ turbulence model is the most widely employed two-equation eddy viscosity model. It is based on the solution of equations for the turbulent kinetic energy and the turbulent dissipation rate. The historic roots of the $\mathrm{k}-\varepsilon$ model reach to the work of Chou (Chou 1945). During the 1970's, various formulations of the model were proposed. The most important contributions were due to Jones and Launder, Launder and Sharma (Launder 1974) as well as due to Launder and Spalding (Launder 1974).

The first approach for the approximate treatment of turbulent flows was presented by Reynolds in 1895. The methodology is based on the decomposition of the flow variables 
into a mean and a fluctuating part Eqn. (1). The governing equations are solved for the mean values, which are the most interesting for engineering applications. The velocity components is substituted by (Blazek 2001).

$$
v_{i}=\bar{v}_{i}+v_{i}^{\prime}
$$

where the mean value is denoted by an overbar and the turbulent fluctuation by a prime (Figure 1).

Appropriate for general turbulence

$$
\bar{v}_{i}=\lim _{N \rightarrow \infty} \frac{1}{N} \sum_{i=1}^{N} v_{i}
$$

Hence, the mean value $\bar{v}_{i}$ still remains a function of time and space coordinates, the average of the fluctuating part is zero, i.e., $\bar{v}_{i}^{\prime}=0$. However, it can be easily seen that $\overline{v_{i}^{\prime} v_{i}^{\prime}} \neq 0$ and $\overline{v_{i}^{\prime} v_{j}^{\prime}} \neq 0$ if both turbulent velocity components are correlated.

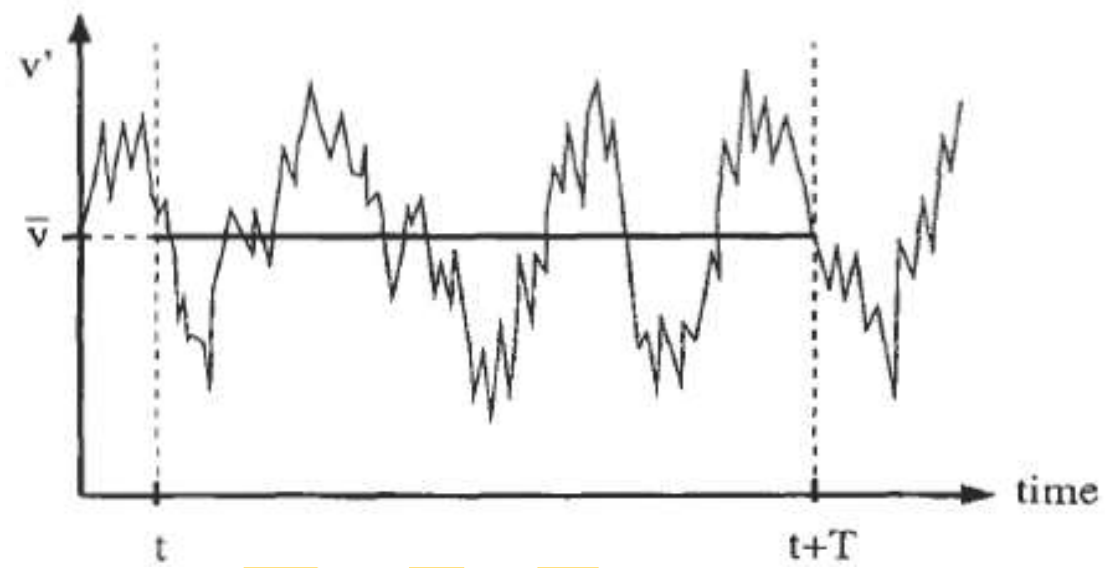

Figure 1. Reynolds averaging illustration of turbulent velocity $\left(\bar{v}, v^{\prime}\right)$

The sum of the normal stresses divided by density defines the turbulent kinetic energy, i.e.,

$$
k=\frac{1}{2} \overline{v_{i}^{\prime} v_{i}^{\prime}}=\frac{1}{2}\left[\overline{\left(v_{1}^{\prime}\right)^{2}}+\overline{\left(v_{2}^{\prime}\right)^{2}}+\overline{\left(v_{3}^{\prime}\right)^{2}}\right]
$$

The differential form of a low Reynolds number k- $\varepsilon$ model can be written as

$$
\begin{aligned}
& \frac{\partial(\rho k)}{\partial t}+\frac{\partial\left(\rho k v_{j}\right)}{\partial x_{j}}=\frac{\partial}{\partial x_{j}}\left[\left(\mu_{L}+\frac{\mu_{T}}{\sigma_{k}}\right) \frac{\partial k}{\partial x_{j}}\right]+\tau_{i j}^{F} S_{i j}-\rho \varepsilon \\
& \frac{\partial(\rho \varepsilon)}{\partial t}+\frac{\partial\left(\rho v_{j} \varepsilon\right)}{\partial x_{j}}=\frac{\partial}{\partial x_{j}}\left[\left(\mu_{L}+\frac{\mu_{T}}{\sigma_{k}}\right) \frac{\partial \varepsilon}{\partial x_{j}}\right]+C_{\varepsilon 1} f_{\varepsilon 1} \frac{\varepsilon}{k} \tau_{i j}^{F} S_{i j}-C_{\varepsilon 2} f_{\varepsilon 2} \rho \frac{\varepsilon^{2}}{k}+\phi_{\varepsilon}
\end{aligned}
$$

In case where the turbulent flow is non-homogeneous two-phase, the Eqn. (3) is not suitable application unless we assume that the flow is homogeneous flow or one phase flow, because the velocities of non-homogeneous two-phase flow include velocity of the gas phase and velocity of the second phase. So that, Eqns. $(3) \div(5)$ must be improved for non-homogeneous two-phase flows. 


\section{GOVERNING EQUATIONS AND SOLUTION METHOD}

\subsection{Governing equations}

The flow-system equations of two-phase turbulent flows were established by Schraiber et al. as follows:

$$
\left\{\begin{array}{l}
\frac{\partial \rho_{g}}{\partial t}+\sum_{j}\left(\frac{\partial \rho_{g j} v_{g j}}{\partial x_{j}}\right)=0 \\
\frac{\partial \rho_{p}}{\partial t}+\sum_{j}\left(\frac{\partial \rho_{p j} v_{p j}}{\partial x_{j}}\right)=0 \\
\frac{\partial v_{g i}}{\partial t}+\sum_{j} v_{g j} \frac{\partial v_{g i}}{\partial x_{j}}=-\frac{1}{\rho_{g}} \frac{\partial p}{\partial x_{i}}+\frac{1}{\rho_{g}} \sum_{j} \frac{\partial \tau_{g i j}}{\partial x_{j}}-\frac{1}{\rho_{g}} F_{i} \\
\frac{\partial v_{p i}}{\partial t}+\sum_{j} v_{p j} \frac{\partial v_{p i}}{\partial x_{j}}=\frac{1}{\rho_{p}} \sum_{j} \frac{\partial \tau_{p i j}}{\partial x_{j}}+\frac{1}{\rho_{p}} F_{i}
\end{array}\right.
$$

Following Erlebacher et al. (1992), the flow variables are decomposed into mean and turbulent fluctuationvelocities as Eqns. (7)

$$
\begin{aligned}
& v_{g}=\bar{v}_{g}+v_{g}^{\prime} \\
& v_{p}=\bar{v}_{p}+v_{p}^{\prime}
\end{aligned}
$$

where $g$, pare the symbols for the gas phase and the second phase; $i, j=1,2,3$ (according to the coordinates); $v_{g i}, v_{g j}, v_{p i}, v_{p j}$ are the velocity components; $\rho_{\mathrm{g}}, \rho_{\mathrm{p}}$ are the density of the phases; $p$ is the pressure; $\tau_{\mathrm{gij}}, \tau_{\mathrm{p} i j}$ are the stress components of the phases; $F_{i}$ is the interaction force between the phases; and the prime symbol means a turbulent component.

Because the average components always satisfy Eqns. (6), the above system equations for the turbulent components in two-dimension coordinates becomes

$$
\begin{aligned}
& \frac{\partial \rho_{g}}{\partial t}+\frac{\partial\left(\rho_{g} u_{g}^{\prime}\right)}{\partial x}+\frac{\partial\left(\rho_{g} v_{g}^{\prime}\right)}{\partial y}=0 \\
& \frac{\partial \rho_{p}}{\partial t}+\frac{\partial\left(\rho_{p} u_{p}^{\prime}\right)}{\partial x}+\frac{\partial\left(\rho_{p} v_{p}^{\prime}\right)}{\partial y}=0 \\
& \frac{\partial u_{g}^{\prime}}{\partial t}+\left(u_{g}^{\prime} \frac{\partial \bar{u}_{g}}{\partial x}+v_{g}^{\prime} \frac{\partial \bar{u}_{g}}{\partial y}\right)+\left(\bar{u}_{g} \frac{\partial u_{g}^{\prime}}{\partial x}+\bar{v}_{g} \frac{\partial u_{g}^{\prime}}{\partial y}\right)+\left(u_{g}^{\prime} \frac{\partial u_{g}^{\prime}}{\partial x}+v_{g}^{\prime} \frac{\partial u_{g}^{\prime}}{\partial y}\right) \\
& =-\frac{1}{\rho_{g}} \frac{\partial p^{\prime}}{\partial x}+\frac{1}{\rho_{g}}\left(\frac{\partial \tau_{g x x}^{\prime}}{\partial x}+\frac{\partial \tau_{g x y}^{\prime}}{\partial y}\right)-\frac{1}{\rho_{g}} F_{x}^{\prime}
\end{aligned}
$$




$$
\begin{aligned}
& \frac{\partial v_{g}^{\prime}}{\partial t}+\left(u_{g}^{\prime} \frac{\partial \bar{v}_{g}}{\partial x}+v_{g}^{\prime} \frac{\partial \bar{v}_{g}}{\partial y}\right)+\left(\bar{u}_{g} \frac{\partial v_{g}^{\prime}}{\partial x}+\bar{v}_{g} \frac{\partial v_{g}^{\prime}}{\partial y}\right)+\left(u_{g}^{\prime} \frac{\partial v_{g}^{\prime}}{\partial x}+v_{g}^{\prime} \frac{\partial v_{g}^{\prime}}{\partial y}\right) \\
& =-\frac{1}{\rho_{g}} \frac{\partial p^{\prime}}{\partial x}+\frac{1}{\rho_{g}}\left(\frac{\partial \tau_{g x y}^{\prime}}{\partial x}+\frac{\partial \tau_{g y y}^{\prime}}{\partial y}\right)-\frac{1}{\rho_{g}} F_{y}^{\prime}
\end{aligned}
$$

$$
\begin{aligned}
& \frac{\partial u_{p}^{\prime}}{\partial t}+\left(u_{p}^{\prime} \frac{\partial \bar{u}_{p}}{\partial x}+v_{p}^{\prime} \frac{\partial \bar{u}_{p}}{\partial y}\right)+\left(\bar{u}_{p} \frac{\partial u_{p}^{\prime}}{\partial x}+\bar{v}_{p} \frac{\partial u_{p}^{\prime}}{\partial y}\right)+\left(u_{p}^{\prime} \frac{\partial u_{p}^{\prime}}{\partial x}+v_{p}^{\prime} \frac{\partial u_{p}^{\prime}}{\partial y}\right) \\
& =\frac{1}{\rho_{p}}\left(\frac{\partial \tau_{p x x}^{\prime}}{\partial x}+\frac{\partial \tau_{p x y}^{\prime}}{\partial y}\right)+\frac{1}{\rho_{p}} F_{x}^{\prime}
\end{aligned}
$$

$$
\begin{aligned}
& \frac{\partial v_{p}^{\prime}}{\partial t}+\left(u_{p}^{\prime} \frac{\partial \bar{v}_{p}}{\partial x}+v_{p}^{\prime} \frac{\partial \bar{v}_{p}}{\partial y}\right)+\left(\bar{u}_{p} \frac{\partial v_{p}^{\prime}}{\partial x}+\bar{v}_{p} \frac{\partial v_{p}^{\prime}}{\partial y}\right)+\left(u_{p}^{\prime} \frac{\partial v_{p}^{\prime}}{\partial x}+v_{p}^{\prime} \frac{\partial v_{p}^{\prime}}{\partial y}\right) \\
& =\frac{1}{\rho_{p}}\left(\frac{\partial \tau_{p x y}^{\prime}}{\partial x}+\frac{\partial \tau_{p y y}^{\prime}}{\partial y}\right)+\frac{1}{\rho_{p}} F_{y}^{\prime}
\end{aligned}
$$

Using Eqns. (8) $\div(13)$ for the turbulent components, we can set up equations for the turbulent kinetic energy $\left(k_{\mathrm{g}}-k_{\mathrm{p}}\right)$ and the turbulent dissipation rate $\left(\varepsilon_{\mathrm{g}}-\varepsilon_{\mathrm{p}}\right)$. Because the equations $k_{\mathrm{g}}-\varepsilon_{\mathrm{g}}$ of the gas phase have already been developed by other authors (Launder 1974; Blazek 2001), so in this paper we just have to write equations for the turbulent kinetic energy $k_{p}$ and turbulent dissipation rate $\varepsilon_{\mathrm{p}}$ of the second phase.

$$
\begin{aligned}
& \rho_{g} \frac{\partial k_{g}}{\partial t}+\rho_{g} u_{g} \frac{\partial k_{g}}{\partial x}+\rho_{g} v_{g} \frac{\partial k_{g}}{\partial y}=\frac{\partial}{\partial y}\left[\frac{\mu_{g}}{\sigma_{k}}\left(\frac{\partial k_{g}}{\partial y}\right)\right]+\mu_{g}\left(\frac{\partial u_{g}}{\partial y}\right)^{2}-\rho_{g} \varepsilon_{g}-\varepsilon^{*}(14) \\
& \rho_{g} \frac{\partial \varepsilon_{g}}{\partial t}+\rho_{g} u_{g} \frac{\partial \varepsilon_{g}}{\partial x}+\rho_{g} v_{g} \frac{\partial \varepsilon_{g}}{\partial y}=\frac{\partial}{\partial y}\left[\frac{\mu_{g}}{\sigma_{\varepsilon}}\left(\frac{\partial \varepsilon_{g}}{\partial y}\right)\right]+C_{\varepsilon 1} \mu_{g} \frac{\varepsilon_{g}}{k_{g}}\left(\frac{\partial v_{g}}{\partial y}\right)^{2}-C_{\varepsilon 2} \frac{\varepsilon_{g}^{2}}{k_{g}}-\phi_{\varepsilon}(15)
\end{aligned}
$$

\subsection{Turbulent kinetic energy equation of the second phase $k_{p}$}

Multiplying Eqn. (12) by the turbulent fluctuation of velocity $u_{p}^{\prime}$, and Eqn. (13) by the turbulent fluctuation of velocity $v^{\prime}$, we get

$$
\begin{aligned}
& u_{p}^{\prime} \frac{\partial u_{p}^{\prime}}{\partial t}+\left(u_{p}^{\prime} u_{p}^{\prime} \frac{\partial \bar{u}_{p}}{\partial x}+u_{p}^{\prime} v_{p}^{\prime} \frac{\partial \bar{u}_{p}}{\partial y}\right)+\left(u_{p}^{\prime} \bar{u}_{p} \frac{\partial u_{p}^{\prime}}{\partial x}+u_{p}^{\prime} \bar{v}_{p} \frac{\partial u_{p}^{\prime}}{\partial y}\right)+\left(u_{p}^{\prime} u_{p}^{\prime} \frac{\partial u_{p}^{\prime}}{\partial x}+u_{p}^{\prime} v_{p} \frac{\partial u_{p}^{\prime}}{\partial y}\right) \\
& =\frac{1}{\rho_{p}}\left(u_{p}^{\prime} \frac{\partial \tau_{p x x}^{\prime}}{\partial x}+u_{p}^{\prime} \frac{\partial \tau_{p x y}^{\prime}}{\partial y}\right)+\frac{1}{\rho_{p}} u_{p}^{\prime} F_{x}^{\prime}
\end{aligned}
$$




$$
\begin{aligned}
& v_{p}^{\prime} \frac{\partial v_{p}^{\prime}}{\partial t}+\left(v_{p}^{\prime} u_{p}^{\prime} \frac{\partial \bar{v}_{p}}{\partial x}+v_{p}^{\prime} v_{p}^{\prime} \frac{\partial \bar{v}_{p}}{\partial y}\right)+\left(v_{p}^{\prime} \bar{u}_{p} \frac{\partial v_{p}^{\prime}}{\partial x}+v_{p}^{\prime} \bar{v}_{p} \frac{\partial v_{p}^{\prime}}{\partial y}\right)+\left(v_{p}^{\prime} u_{p}^{\prime} \frac{\partial v_{p}^{\prime}}{\partial x}+v_{p}^{\prime} v_{p}^{\prime} \frac{\partial v_{p}^{\prime}}{\partial y}\right) \\
& =\frac{1}{\rho_{p}}\left(v_{p}^{\prime} \frac{\partial \tau_{p x y}^{\prime}}{\partial x}+v_{p}^{\prime} \frac{\partial \tau_{p y y}^{\prime}}{\partial y}\right)+\frac{1}{\rho_{p}} v_{p}^{\prime} F_{y}^{\prime}
\end{aligned}
$$

Adding both sides of Eqn. (14) and Eqn. (15) we get the Eqn. (16)

$$
\begin{aligned}
& \rho_{p}\left(u_{p}^{\prime} \frac{\partial u_{p}^{\prime}}{\partial t}+v_{p}^{\prime} \frac{\partial v_{p}^{\prime}}{\partial t}\right) \\
& +\rho_{p}\left(u_{p}^{\prime} u_{p}^{\prime} \frac{\partial \bar{u}_{p}}{\partial x}+u_{p}^{\prime} \bar{u}_{p} \frac{\partial u_{p}^{\prime}}{\partial x}+u_{p}^{\prime} u_{p}^{\prime} \frac{\partial u_{p}^{\prime}}{\partial x}+v_{p}^{\prime} u_{p}^{\prime} \frac{\partial \bar{v}_{p}}{\partial x}+v_{p}^{\prime} \bar{u}_{p} \frac{\partial v_{p}^{\prime}}{\partial x}+v_{p}^{\prime} u_{p}^{\prime} \frac{\partial v_{p}^{\prime}}{\partial x}\right) \\
& +\rho_{p}\left(u_{p}^{\prime} v_{p}^{\prime} \frac{\partial \bar{u}_{p}}{\partial y}+u_{p}^{\prime} \bar{v}_{p} \frac{\partial u_{p}^{\prime}}{\partial y}+u_{p}^{\prime} v_{p}^{\prime} \frac{\partial u_{p}^{\prime}}{\partial y}+v_{p}^{\prime} v_{p}^{\prime} \frac{\partial \bar{v}_{p}}{\partial y}+v_{p}^{\prime} \bar{v}_{p} \frac{\partial v_{p}^{\prime}}{\partial y}+v_{p}^{\prime} v_{p}^{\prime} \frac{\partial v_{p}^{\prime}}{\partial y}\right) \\
& =\left[\left(u_{p}^{\prime} \frac{\partial \tau_{p x x}^{\prime}}{\partial x}+u_{p}^{\prime} \frac{\partial \tau_{p x y}^{\prime}}{\partial y}\right)+\left(v_{p}^{\prime} \frac{\partial \tau_{p x y}^{\prime}}{\partial x}+v_{p}^{\prime} \frac{\partial \tau_{p y y}^{\prime}}{\partial y}\right)\right]+\left(u_{p}^{\prime} F_{x}^{\prime}+v_{p}^{\prime} F_{y}^{\prime}\right)
\end{aligned}
$$

The turbulent kinetic energy $k_{p}$ in this equation is defined as Eqn. (17)

$$
k_{p}=\frac{1}{2}\left(u_{p}^{\prime 2}+v_{p}^{2}\right)
$$

So that, the first expression in the left-hand side of Eqn. (16) can be developed

$$
\rho_{p}\left(u_{p}^{\prime} \frac{\partial u_{p}^{\prime}}{\partial t}+v_{p}^{\prime} \frac{\partial v_{p}^{\prime}}{\partial t}\right)=\rho_{p} \frac{\partial}{\partial t}\left[\frac{1}{2}\left(u_{p}^{\prime 2}+v_{p}^{\prime 2}\right)\right]=\rho_{p} \frac{\partial k_{p}}{\partial t}
$$

The second expression in the left-hand side of Eqn. (16) can be developed

$$
\begin{aligned}
& \rho_{p}\left(u_{p}^{\prime} u_{p}^{\prime} \frac{\partial \bar{u}_{p}}{\partial x}+u_{p}^{\prime} \bar{u}_{p} \frac{\partial u_{p}^{\prime}}{\partial x}+u_{p}^{\prime} u_{p}^{\prime} \frac{\partial u_{p}^{\prime}}{\partial x}+v_{p}^{\prime} u_{p}^{\prime} \frac{\partial \bar{v}_{p}}{\partial x}+v_{p}^{\prime} \bar{u}_{p} \frac{\partial v_{p}^{\prime}}{\partial x}+v_{p}^{\prime} u_{p}^{\prime} \frac{\partial v_{p}^{\prime}}{\partial x}\right) \\
& =\rho_{p}\left[u_{p}^{\prime}\left(u_{p}^{\prime} \frac{\partial \bar{u}_{p}}{\partial x}+v_{p}^{\prime} \frac{\partial \bar{v}_{p}}{\partial x}\right)\right]+\rho_{p}\left[\frac{\left(u_{p}^{\prime}+\bar{u}_{p}\right)}{2} \frac{\partial\left(u_{p}^{\prime 2}+v_{p}^{\prime 2}\right)}{\partial x}\right]=\rho_{p} u_{p} \frac{\partial k_{p}}{\partial x}
\end{aligned}
$$

The third expression in the left-hand side of Eqn. (16) can be developed

$$
\begin{aligned}
& \rho_{p}\left(u_{p}^{\prime} v_{p}^{\prime} \frac{\partial \bar{u}_{p}}{\partial y}+u_{p}^{\prime} \bar{v}_{p} \frac{\partial u_{p}^{\prime}}{\partial y}+u_{p}^{\prime} v_{p}^{\prime} \frac{\partial u_{p}^{\prime}}{\partial y}+v_{p}^{\prime} v_{p}^{\prime} \frac{\partial \bar{v}_{p}}{\partial y}+v_{p}^{\prime} \bar{v}_{p} \frac{\partial v_{p}^{\prime}}{\partial y}+v_{p}^{\prime} v_{p}^{\prime} \frac{\partial v_{p}^{\prime}}{\partial y}\right) \\
& =\rho_{p}\left[v_{p}^{\prime}\left(u_{p}^{\prime} \frac{\partial \bar{u}_{p}}{\partial y}+v_{p}^{\prime} \frac{\partial \bar{v}_{p}}{\partial y}\right)\right]+\rho_{p}\left[\frac{v_{p}}{2} \frac{\partial\left(u_{p}^{\prime 2}+v_{p}^{\prime 2}\right)}{\partial y}\right]=\rho_{p} v_{p} \frac{\partial k_{p}}{\partial y}
\end{aligned}
$$

The first expression in the right-hand side of Eqn. (16) can be developed 
Engineering International, Volume 2, No 1 (2014)

$$
\begin{aligned}
& {\left[\left(u_{p}^{\prime} \frac{\partial \tau_{p x x}^{\prime}}{\partial x}+u_{p}^{\prime} \frac{\partial \tau_{p x y}^{\prime}}{\partial y}\right)+\left(v_{p}^{\prime} \frac{\partial \tau_{p x y}^{\prime}}{\partial x}+v_{p}^{\prime} \frac{\partial \tau_{p y y}^{\prime}}{\partial y}\right)\right]} \\
& =\left[\left(u_{p}^{\prime} \frac{\partial \tau_{p x x}^{\prime}}{\partial x}+v_{p}^{\prime} \frac{\partial \tau_{p x y}^{\prime}}{\partial x}\right)+\left(u_{p}^{\prime} \frac{\partial \tau_{p x y}^{\prime}}{\partial y}+v_{p}^{\prime} \frac{\partial \tau_{p y y}^{\prime}}{\partial y}\right)\right] \\
& =\left[\frac{\partial}{\partial x}\left(u_{p}^{\prime} \tau_{p x x}^{\prime}\right)+\frac{\partial}{\partial x}\left(v_{p}^{\prime} \tau_{p x y}^{\prime}\right)+\frac{\partial}{\partial y}\left(u_{p}^{\prime} \tau_{p x y}^{\prime}\right)+\frac{\partial}{\partial y}\left(v_{p}^{\prime} \tau_{p y y}^{\prime}\right)\right] \\
& -\left[\frac{\partial u_{p}^{\prime}}{\partial x} \tau_{p x x}^{\prime}+\frac{\partial v_{p}^{\prime}}{\partial x} \tau_{p x y}^{\prime}+\frac{\partial u_{p}^{\prime}}{\partial y} \tau_{p x y}^{\prime}+\frac{\partial v_{p}^{\prime}}{\partial y} \tau_{p y y}^{\prime}\right]
\end{aligned}
$$

The components of the viscous stress tensor are defined by the relations $[8,9]$

$$
\begin{aligned}
\tau_{p x y} & =\mu_{p}\left(\frac{\partial v^{\prime}{ }_{p}}{\partial x}+\frac{\partial u^{\prime}{ }_{p}}{\partial y}\right) \\
\tau_{p x x} & =\lambda . \nabla U+2 \mu_{p} \frac{\partial u^{\prime}{ }_{p}}{\partial x} \\
\tau^{\prime}{ }_{p y y} & =\lambda . \nabla U+2 \mu_{p} \frac{\partial v^{\prime}{ }_{p}}{\partial y}
\end{aligned}
$$

Substituting Eqns. (22) into Eqn. (21) and rearranging, we get the following equation:

$$
\begin{aligned}
& {\left[\left(u_{p}^{\prime} \frac{\partial \tau_{p x x}^{\prime}}{\partial x}+u_{p}^{\prime} \frac{\partial \tau_{p x y}^{\prime}}{\partial y}\right)+\left(v_{p}^{\prime} \frac{\partial \tau_{p x y}^{\prime}}{\partial x}+v_{p}^{\prime} \frac{\partial \tau_{p y y}^{\prime}}{\partial y}\right)\right]} \\
& =\mu_{p}\left[2 \frac{\partial}{\partial x}\left(u_{p}^{\prime} \frac{\partial u_{p}^{\prime}}{\partial x}\right)+2 \frac{\partial}{\partial y}\left(v_{p}^{\prime} \frac{\partial v_{p}^{\prime}}{\partial y}\right)+\frac{\partial}{\partial x}\left(v_{p}^{\prime} \frac{\partial v_{p}^{\prime}}{\partial x}+v_{p}^{\prime} \frac{\partial u_{p}^{\prime}}{\partial y}\right)\right. \\
& \left.+\frac{\partial}{\partial y}\left(u_{p}^{\prime} \frac{\partial v_{p}^{\prime}}{\partial x}+u_{p}^{\prime} \frac{\partial u_{p}^{\prime}}{\partial y}\right)\right]-\mu_{p}\left(3 \frac{\partial u_{p}^{\prime}}{\partial x} \frac{\partial v_{p}^{\prime}}{\partial x}+\frac{\partial u_{p}^{\prime}}{\partial x} \frac{\partial u_{p}^{\prime}}{\partial y}+\frac{\partial v_{p}^{\prime}}{\partial x} \frac{\partial v_{p}^{\prime}}{\partial y}+3 \frac{\partial u_{p}^{\prime}}{\partial y} \frac{\partial v_{p}^{\prime}}{\partial y}\right)
\end{aligned}
$$

The first expression in the right-hand side of Eqn. (23) can be developed

$$
\begin{aligned}
& \mu_{p}\left[2 \frac{\partial}{\partial x}\left(u_{p}^{\prime} \frac{\partial u_{p}^{\prime}}{\partial x}\right)+2 \frac{\partial}{\partial y}\left(v_{p}^{\prime} \frac{\partial v_{p}^{\prime}}{\partial y}\right)+\frac{\partial}{\partial x}\left(v_{p}^{\prime} \frac{\partial v_{p}^{\prime}}{\partial x}+v_{p}^{\prime} \frac{\partial u_{p}^{\prime}}{\partial y}\right)+\frac{\partial}{\partial y}\left(u_{p}^{\prime} \frac{\partial v_{p}^{\prime}}{\partial x}+u_{p}^{\prime} \frac{\partial u_{p}^{\prime}}{\partial y}\right)\right] \\
& =\mu_{p}\left[\frac{\partial}{\partial x}\left(u_{p}^{\prime} \frac{\partial u_{p}^{\prime}}{\partial x}+v_{p}^{\prime} \frac{\partial v_{p}^{\prime}}{\partial x}\right)+\frac{\partial}{\partial y}\left(u_{p}^{\prime} \frac{\partial u_{p}^{\prime}}{\partial y}+v_{p}^{\prime} \frac{\partial v_{p}^{\prime}}{\partial y}\right)\right] \\
& +\mu_{p}\left[\frac{\partial}{\partial y}\left(u_{p}^{\prime} \frac{\partial v_{p}^{\prime}}{\partial x}+v_{p}^{\prime} \frac{\partial v_{p}^{\prime}}{\partial y}\right)+\frac{\partial}{\partial x}\left(u_{p}^{\prime} \frac{\partial u_{p}^{\prime}}{\partial x}+v_{p}^{\prime} \frac{\partial u_{p}^{\prime}}{\partial y}\right)\right] \\
& =\frac{\partial}{\partial y}\left[\frac{\mu_{p}}{\sigma_{k}}\left(\frac{\partial k_{p}}{\partial y}\right)\right]+\frac{\partial}{\partial y}\left[k_{p} \mu_{p}\left(\frac{\partial u_{p}}{\partial y}\right)\right]+\mu_{p}\left(\frac{\partial u_{p}}{\partial y}\right)^{2}
\end{aligned}
$$

Using the Kolmogorov formulation for the turbulent dissipation rate $\varepsilon$, the second expression in the right-hand side of Eqn. (23) can be developed 


$$
\mu_{p}\left(3 \frac{\partial u_{p}^{\prime}}{\partial x} \frac{\partial v_{p}^{\prime}}{\partial x}+\frac{\partial u_{p}^{\prime}}{\partial x} \frac{\partial u_{p}^{\prime}}{\partial y}+\frac{\partial v_{p}^{\prime}}{\partial x} \frac{\partial v_{p}^{\prime}}{\partial y}+3 \frac{\partial u_{p}^{\prime}}{\partial y} \frac{\partial v_{p}^{\prime}}{\partial y}\right)=\rho_{p} \varepsilon_{p}
$$

The second expression in the right-hand side of Eqn. (16) can be developed

$$
\frac{1}{\rho_{p}}\left(u_{p}^{\prime} F_{x}^{\prime}+v_{p}^{\prime} F_{y}^{\prime}\right)=\varepsilon^{*}
$$

Substituting Eqns. (18), (19), (20), (24), (25), and (26) into Eqn. (16), we get the following equation:

$$
\begin{aligned}
& \rho_{p} \frac{\partial k_{p}}{\partial t}+\rho_{p} u_{p} \frac{\partial k_{p}}{\partial x}+\rho_{p} v_{p} \frac{\partial k_{p}}{\partial y} \\
& =\frac{\partial}{\partial y}\left[\rho_{p} \frac{\mu_{p}}{\sigma_{k}}\left(\frac{\partial k_{p}}{\partial y}\right)\right]+\frac{\partial}{\partial y}\left[k_{p} \mu_{p}\left(\frac{\partial u_{p}}{\partial y}\right)\right]+\mu_{p}\left(\frac{\partial u_{p}}{\partial y}\right)^{2}-\rho_{p} \varepsilon_{p}+\varepsilon^{*}
\end{aligned}
$$

Eqn. (27) is the turbulent kinetic energy of the second phase of non-homogeneous two-phase flows.

\subsection{Turbulent dissipation rate equation of the second phase Ep}

The equations of turbulent dissipation rate were established by Schraiber et al. as follows:

$$
\begin{aligned}
& \frac{\partial}{\partial t}\left(\mu_{p} \frac{\partial u_{p}^{\prime}}{\partial y} \frac{\partial u_{p}^{\prime}}{\partial y}\right)=2 \mu_{p} \frac{\partial u_{p}^{\prime}}{\partial y} \frac{\partial}{\partial y}\left(\frac{\partial u_{p}^{\prime}}{\partial t}\right) \\
& \frac{\partial}{\partial t}\left(\mu_{p} \frac{\partial v_{p}^{\prime}}{\partial x} \frac{\partial v_{p}^{\prime}}{\partial x}\right)=2 \mu_{p} \frac{\partial v_{p}^{\prime}}{\partial x} \frac{\partial}{\partial x}\left(\frac{\partial v_{p}^{\prime}}{\partial t}\right)
\end{aligned}
$$

Substituting $\partial u_{p}^{\prime} / \partial t, \partial v_{p}^{\prime} / \partial t$ values from Eqns. (12) and (13) into Eqns. (28) and (29), we get the following equations:

$$
\begin{aligned}
& \frac{\partial}{\partial t}\left(\mu_{p} \frac{\partial u_{p}^{\prime}}{\partial y} \frac{\partial u_{p}^{\prime}}{\partial y}\right)=2 \mu_{p} \frac{\partial u_{p}^{\prime}}{\partial y} \frac{\partial}{\partial y}\left[-\left(u_{p}^{\prime} \frac{\partial \bar{u}_{p}}{\partial x}+v_{p}^{\prime} \frac{\partial \bar{u}_{p}}{\partial y}\right)-\left(\bar{u}_{p} \frac{\partial u_{p}^{\prime}}{\partial x}+\bar{v}_{p} \frac{\partial u_{p}^{\prime}}{\partial y}\right)\right. \\
& \left.-\left(u_{p}^{\prime} \frac{\partial u_{p}^{\prime}}{\partial x}+v_{p}^{\prime} \frac{\partial u_{p}^{\prime}}{\partial y}\right)+\frac{1}{\rho_{p}}\left(\frac{\partial \tau_{p x x}^{\prime}}{\partial x}+\frac{\partial \tau_{p x y}^{\prime}}{\partial y}\right)+\frac{1}{\rho_{p}} F_{x}^{\prime}\right] \\
& \frac{\partial}{\partial t}\left(\mu_{p} \frac{\partial v_{p}^{\prime}}{\partial x} \frac{\partial v_{p}^{\prime}}{\partial x}\right)=2 \mu_{p} \frac{\partial v_{p}^{\prime}}{\partial x} \frac{\partial}{\partial x}\left[-\left(u_{p}^{\prime} \frac{\partial \bar{v}_{p}}{\partial x}+v_{p}^{\prime} \frac{\partial \bar{v}_{p}}{\partial y}\right)-\left(\bar{u}_{p} \frac{\partial v_{p}^{\prime}}{\partial x}+\bar{v}_{p} \frac{\partial v_{p}^{\prime}}{\partial y}\right)\right. \\
& \left.-\left(u_{p}^{\prime} \frac{\partial v_{p}^{\prime}}{\partial x}+v_{p}^{\prime} \frac{\partial v_{p}^{\prime}}{\partial y}\right)+\frac{1}{\rho_{p}}\left(\frac{\partial \tau_{p x y}^{\prime}}{\partial x}+\frac{\partial \tau_{p y y}^{\prime}}{\partial y}\right)+\frac{1}{\rho_{p}} F_{y}^{\prime}\right]
\end{aligned}
$$

Substituting Eqn. (22) into Eqns. (30) and (31), we get the following equations: 
Engineering International, Volume 2, No 1 (2014)

$$
\begin{aligned}
& \frac{\partial}{\partial t}\left(\mu_{p} \frac{\partial u_{p}^{\prime}}{\partial y} \frac{\partial u_{p}^{\prime}}{\partial y}\right) \\
& =-2 \mu_{p}\left\{u_{p}^{\prime} \frac{\partial u_{p}^{\prime}}{\partial y} \frac{\partial}{\partial y}\left(\frac{\partial u_{p}^{\prime}}{\partial x}\right)+\bar{v}_{p} \frac{\partial u_{p}^{\prime}}{\partial y} \frac{\partial}{\partial y}\left(\frac{\partial u_{p}^{\prime}}{\partial y}\right)+v_{p}^{\prime} \frac{\partial u_{p}^{\prime}}{\partial y} \frac{\partial}{\partial y}\left(\frac{\partial \bar{u}_{p}}{\partial y}\right)\right. \\
& +v_{p}^{\prime} \frac{\partial u_{p}^{\prime}}{\partial y} \frac{\partial}{\partial y}\left(\frac{\partial u_{p}^{\prime}}{\partial y}\right)+\frac{\partial u_{p}^{\prime}}{\partial y} \frac{\partial \bar{u}_{p}}{\partial y} \frac{\partial v_{p}^{\prime}}{\partial y}+\frac{\partial u_{p}^{\prime}}{\partial y} \frac{\partial u_{p}^{\prime}}{\partial y} \frac{\partial \bar{v}_{p}}{\partial y}+\frac{\partial u_{p}^{\prime}}{\partial y} \frac{\partial u_{p}^{\prime}}{\partial y} \frac{\partial u_{p}^{\prime}}{\partial x} \\
& +\frac{\partial u_{p}^{\prime}}{\partial y} \frac{\partial u_{p}^{\prime}}{\partial y} \frac{\partial v_{p}^{\prime}}{\partial y}+\frac{\partial u_{p}^{\prime}}{\partial y} \frac{\partial}{\partial y} \frac{\partial\left(u_{p}^{\prime} \bar{u}_{p}\right)}{\partial x}-2 \frac{\partial u_{p}^{\prime}}{\partial y} \frac{\partial}{\partial y}\left[\frac{\partial}{\partial y}\left(\frac{\partial u_{p}^{\prime}}{\partial x}\right)\right] \\
& \left.-\frac{\partial u_{p}^{\prime}}{\partial y} \frac{\partial}{\partial y}\left[\frac{\partial}{\partial y}\left(\frac{\partial u_{p}^{\prime}}{\partial y}\right)\right]-\frac{\partial u_{p}^{\prime}}{\partial y} \frac{\partial}{\partial y}\left[\frac{\partial}{\partial y}\left(\frac{\partial v_{p}^{\prime}}{\partial x}\right)\right]-\frac{1}{\rho_{p}} \frac{\partial u_{p}^{\prime}}{\partial y} \frac{\partial F_{x}^{\prime}}{\partial y}\right\} \\
& \frac{\partial}{\partial t}\left(\mu_{p} \frac{\partial v_{p}^{\prime}}{\partial x} \frac{\partial v_{p}^{\prime}}{\partial x}\right) \\
& =-2 \mu_{p}\left\{u_{p}^{\prime} \frac{\partial v_{p}^{\prime}}{\partial x} \frac{\partial}{\partial x}\left(\frac{\partial v_{p}^{\prime}}{\partial x}\right)+\bar{u}_{p} \frac{\partial v_{p}^{\prime}}{\partial x} \frac{\partial}{\partial x}\left(\frac{\partial v_{p}^{\prime}}{\partial x}\right)+u_{p}^{\prime} \frac{\partial v_{p}^{\prime}}{\partial x} \frac{\partial}{\partial x}\left(\frac{\partial \bar{v}_{p}}{\partial x}\right)\right. \\
& +v_{p}^{\prime} \frac{\partial v_{p}^{\prime}}{\partial x} \frac{\partial}{\partial x}\left(\frac{\partial v_{p}^{\prime}}{\partial y}\right)+\frac{\partial v_{p}^{\prime}}{\partial x} \frac{\partial \bar{v}_{p}}{\partial x} \frac{\partial u_{p}^{\prime}}{\partial x}+\frac{\partial v_{p}^{\prime}}{\partial x} \frac{\partial v_{p}^{\prime}}{\partial x} \frac{\partial \bar{u}_{p}}{\partial x}+\frac{\partial v_{p}^{\prime}}{\partial x} \frac{\partial v_{p}^{\prime}}{\partial x} \frac{\partial v_{p}^{\prime}}{\partial y} \\
& +\frac{\partial v_{p}^{\prime}}{\partial x} \frac{\partial v_{p}^{\prime}}{\partial x} \frac{\partial u_{p}^{\prime}}{\partial x}+\frac{\partial v_{p}^{\prime}}{\partial x} \frac{\partial}{\partial x} \frac{\partial\left(v_{p}^{\prime} \bar{v}_{p}\right)}{\partial y}-2 \frac{\partial v_{p}^{\prime}}{\partial x} \frac{\partial}{\partial x}\left[\frac{\partial}{\partial y}\left(\frac{\partial v_{p}^{\prime}}{\partial y}\right)\right] \\
& \left.-\frac{\partial v_{p}^{\prime}}{\partial x} \frac{\partial}{\partial x}\left[\frac{\partial}{\partial x}\left(\frac{\partial v_{p}^{\prime}}{\partial x}\right)\right]-\frac{\partial v_{p}^{\prime}}{\partial x} \frac{\partial}{\partial x}\left[\frac{\partial}{\partial x}\left(\frac{\partial u_{p}^{\prime}}{\partial y}\right)\right]-\frac{1}{\rho_{p}} \frac{\partial v_{p}^{\prime}}{\partial x} \frac{\partial F_{y}^{\prime}}{\partial x}\right\}
\end{aligned}
$$

Adding both sides of Eqn. (32) and Eqn. (33) and rearranging, we get the equation 
Engineering International, Volume 2, No 1 (2014)

$$
\begin{aligned}
& \frac{\partial}{\partial t}\left(\mu_{p} \frac{\partial u_{p}^{\prime}}{\partial y} \frac{\partial u_{p}^{\prime}}{\partial y}\right)+\frac{\partial}{\partial t}\left(\mu_{p} \frac{\partial v_{p}^{\prime}}{\partial x} \frac{\partial v_{p}^{\prime}}{\partial x}\right) \\
& =-2 \mu_{p}\left\{\left[u_{p}^{\prime} \frac{\partial v_{p}^{\prime}}{\partial x} \frac{\partial}{\partial x}\left(\frac{\partial v_{p}^{\prime}}{\partial x}\right)+\bar{u}_{p} \frac{\partial v_{p}^{\prime}}{\partial x} \frac{\partial}{\partial x}\left(\frac{\partial v_{p}^{\prime}}{\partial x}\right)+u_{p}^{\prime} \frac{\partial v_{p}^{\prime}}{\partial x} \frac{\partial}{\partial x}\left(\frac{\partial v_{p}}{\partial x}\right)\right]\right. \\
& +\left[\bar{v}_{p} \frac{\partial u_{p}^{\prime}}{\partial y} \frac{\partial}{\partial y}\left(\frac{\partial u_{p}^{\prime}}{\partial y}\right)+v_{p}^{\prime} \frac{\partial u_{p}^{\prime}}{\partial y} \frac{\partial}{\partial y}\left(\frac{\partial \bar{u}_{p}}{\partial y}\right)+v_{p}^{\prime} \frac{\partial u_{p}^{\prime}}{\partial y} \frac{\partial}{\partial y}\left(\frac{\partial u_{p}^{\prime}}{\partial y}\right)\right] \\
& +\left[u_{p}^{\prime} \frac{\partial u_{p}^{\prime}}{\partial y} \frac{\partial}{\partial y}\left(\frac{\partial u_{p}^{\prime}}{\partial x}\right)+v_{p}^{\prime} \frac{\partial v_{p}^{\prime}}{\partial x} \frac{\partial}{\partial x}\left(\frac{\partial v_{p}^{\prime}}{\partial y}\right)+\frac{\partial u_{p}^{\prime}}{\partial y} \frac{\partial}{\partial y} \frac{\partial\left(u_{p}^{\prime} \overline{u_{p}}\right)}{\partial x}+\frac{\partial v_{p}^{\prime}}{\partial x} \frac{\partial}{\partial x} \frac{\partial\left(v_{p}^{\prime} \bar{v}_{p}\right)}{\partial y}\right] \\
& +\left[\frac{\partial u_{p}^{\prime}}{\partial y} \frac{\partial u_{p}^{\prime}}{\partial y} \frac{\partial u_{p}^{\prime}}{\partial x}+\frac{\partial u_{p}^{\prime}}{\partial y} \frac{\partial u_{p}^{\prime}}{\partial y} \frac{\partial v_{p}^{\prime}}{\partial y}+\frac{\partial v_{p}^{\prime}}{\partial x} \frac{\partial v_{p}^{\prime}}{\partial x} \frac{\partial \bar{u}_{p}}{\partial x}+\frac{\partial v_{p}^{\prime}}{\partial x} \frac{\partial v_{p}^{\prime}}{\partial x} \frac{\partial v_{p}^{\prime}}{\partial y}\right] \\
& +\left[2 \frac { \partial u _ { p } ^ { \prime } } { \partial y } \frac { \partial } { \partial y } \left[\frac{\partial}{\partial y}\left(\frac{\partial u_{p}^{\prime}}{\partial x} \frac{\partial \overline{v_{p}}}{\partial y}+\frac{\partial v_{p}^{\prime}}{\partial x} \frac{\partial \overline{v_{p}}}{\partial x} \frac{\partial u_{p}^{\prime}}{\partial x}+\frac{\partial v_{p}^{\prime}}{\partial x} \frac{\partial v_{p}^{\prime}}{\partial x} \frac{\partial u_{p}^{\prime}}{\partial x}\right]+2 \frac{\partial v_{p}^{\prime}}{\partial x} \frac{\partial}{\partial x}\left[\frac{\partial}{\partial y}\left(\frac{\partial v_{p}^{\prime}}{\partial y}\right)\right]+\frac{\partial v_{p}^{\prime}}{\partial x} \frac{\partial}{\partial x}\left[\frac{\partial}{\partial x}\left(\frac{\partial v_{p}^{\prime}}{\partial x}\right)\right]\right.\right. \\
& \left.+\frac{\partial v_{p}^{\prime}}{\partial x} \frac{\partial}{\partial x}\left[\frac{\partial}{\partial x}\left(\frac{\partial u_{p}^{\prime}}{\partial y}\right)\right]+\frac{\partial u_{p}^{\prime}}{\partial y} \frac{\partial}{\partial y}\left[\frac{\partial}{\partial y}\left(\frac{\partial u_{p}^{\prime}}{\partial y}\right)\right]+\frac{\partial u_{p}^{\prime}}{\partial y} \frac{\partial}{\partial y}\left[\frac{\partial}{\partial y}\left(\frac{\partial v_{p}^{\prime}}{\partial x}\right)\right]\right] \\
& \left.+\left[\frac{1}{\rho_{p}} \frac{\partial u_{p}^{\prime}}{\partial y} \frac{\partial F_{x}^{\prime}}{\partial y}+\frac{1}{\rho_{p}} \frac{\partial v_{p}^{\prime}}{\partial x} \frac{\partial F_{y}^{\prime}}{\partial x}\right]\right\}
\end{aligned}
$$

The expression in the left-hand side of Eqn. (34) is the turbulent dissipation rate:

$$
\frac{\partial}{\partial t}\left(\mu_{p} \frac{\partial u_{p}^{\prime}}{\partial y} \frac{\partial u_{p}^{\prime}}{\partial y}\right)+\frac{\partial}{\partial t}\left(\mu_{p} \frac{\partial v_{p}^{\prime}}{\partial x} \frac{\partial v_{p}^{\prime}}{\partial x}\right)=\rho_{p} \frac{\partial \varepsilon_{p}}{\partial t}
$$

The first expression in the right-hand side of Eqn. (34) can be developed

$$
2 \mu_{p}\left[\bar{u}_{p} \frac{\partial v_{p}^{\prime}}{\partial x} \frac{\partial}{\partial x}\left(\frac{\partial v_{p}^{\prime}}{\partial x}\right)+u_{p}^{\prime} \frac{\partial v_{p}^{\prime}}{\partial x} \frac{\partial}{\partial x}\left(\frac{\partial \bar{v}_{p}}{\partial x}\right)+u_{p}^{\prime} \frac{\partial v_{p}^{\prime}}{\partial x} \frac{\partial}{\partial x}\left(\frac{\partial v_{p}^{\prime}}{\partial x}\right)\right]=\rho_{p} u_{p} \frac{\partial \varepsilon_{p}}{\partial x}
$$

The second expression in the right-hand side of Eqn. (34) can be developed

$$
2 \mu_{p}\left[\bar{v}_{p} \frac{\partial u_{p}^{\prime}}{\partial y} \frac{\partial}{\partial y}\left(\frac{\partial u_{p}^{\prime}}{\partial y}\right)+v_{p}^{\prime} \frac{\partial u_{p}^{\prime}}{\partial y} \frac{\partial}{\partial y}\left(\frac{\partial \bar{u}_{p}}{\partial y}\right)+v_{p}^{\prime} \frac{\partial u_{p}^{\prime}}{\partial y} \frac{\partial}{\partial y}\left(\frac{\partial u_{p}^{\prime}}{\partial y}\right)\right]=\rho_{p} v_{p} \frac{\partial \varepsilon_{p}}{\partial y}
$$

The third expression in the right-hand side of Eqn. (34) can be developed 


$$
\begin{aligned}
& 2 \mu_{p}\left[u_{p}^{\prime} \frac{\partial u_{p}^{\prime}}{\partial y} \frac{\partial}{\partial y}\left(\frac{\partial u_{p}^{\prime}}{\partial x}\right)+v_{p}^{\prime} \frac{\partial v_{p}^{\prime}}{\partial x} \frac{\partial}{\partial x}\left(\frac{\partial v_{p}^{\prime}}{\partial y}\right)+\frac{\partial u_{p}^{\prime}}{\partial y} \frac{\partial}{\partial y} \frac{\partial\left(u_{p}^{\prime} \bar{u}_{p}\right)}{\partial x}+\frac{\partial v_{p}^{\prime}}{\partial x} \frac{\partial}{\partial x} \frac{\partial\left(v_{p}^{\prime} \bar{v}_{p}\right)}{\partial y}\right] \\
& =\frac{\partial}{\partial y}\left[\frac{\mu_{p}}{\sigma_{\varepsilon}}\left(\frac{\partial \varepsilon_{p}}{\partial y}\right)\right]
\end{aligned}
$$

The fourth expression in the right-hand side of Eqn. (34) can be developed

$$
2 \mu_{p}\left[\frac{\partial u_{p}^{\prime}}{\partial y} \frac{\partial u_{p}^{\prime}}{\partial y} \frac{\partial u_{p}^{\prime}}{\partial x}+\frac{\partial u_{p}^{\prime}}{\partial y} \frac{\partial u_{p}^{\prime}}{\partial y} \frac{\partial v_{p}^{\prime}}{\partial y}+\frac{\partial v_{p}^{\prime}}{\partial x} \frac{\partial v_{p}^{\prime}}{\partial x} \frac{\partial v_{p}^{\prime}}{\partial y}+\frac{\partial v_{p}^{\prime}}{\partial x} \frac{\partial v_{p}^{\prime}}{\partial x} \frac{\partial u_{p}^{\prime}}{\partial x}\right]=C_{\varepsilon 2} \frac{\varepsilon_{p}^{2}}{k_{p}}
$$

The fifth expression in the right-hand side of Eqn. (34) can be developed

$$
2 \mu_{p}\left[\frac{\partial u_{p}^{\prime}}{\partial y} \frac{\partial \bar{u}_{p}}{\partial y} \frac{\partial v_{p}^{\prime}}{\partial y}+\frac{\partial u_{p}^{\prime}}{\partial y} \frac{\partial u_{p}^{\prime}}{\partial y} \frac{\partial \bar{v}_{p}}{\partial y}+\frac{\partial v_{p}^{\prime}}{\partial x} \frac{\partial \bar{v}_{p}}{\partial x} \frac{\partial u_{p}^{\prime}}{\partial x}+\frac{\partial v_{p}^{\prime}}{\partial x} \frac{\partial v_{p}^{\prime}}{\partial x} \frac{\partial \bar{u}_{p}}{\partial x}\right]=\mu_{p} C_{\varepsilon 1} \frac{\varepsilon_{p}}{k_{p}}\left(\frac{\partial u_{p}}{\partial y}\right)^{2}
$$

The sixth expression in the right-hand side of Eqn. (34) can be developed

$$
\begin{aligned}
& 2 \mu_{p}\left\{2 \frac{\partial u_{p}^{\prime}}{\partial y} \frac{\partial}{\partial y}\left[\frac{\partial}{\partial y}\left(\frac{\partial u_{p}^{\prime}}{\partial x}\right)\right]+2 \frac{\partial v_{p}^{\prime}}{\partial x} \frac{\partial}{\partial x}\left[\frac{\partial}{\partial y}\left(\frac{\partial v_{p}^{\prime}}{\partial y}\right)\right]+\frac{\partial v_{p}^{\prime}}{\partial x} \frac{\partial}{\partial x}\left[\frac{\partial}{\partial x}\left(\frac{\partial v_{p}^{\prime}}{\partial x}\right)\right]\right. \\
& \left.+\frac{\partial v_{p}^{\prime}}{\partial x} \frac{\partial}{\partial x}\left[\frac{\partial}{\partial x}\left(\frac{\partial u_{p}^{\prime}}{\partial y}\right)\right]+\frac{\partial u_{p}^{\prime}}{\partial y} \frac{\partial}{\partial y}\left[\frac{\partial}{\partial y}\left(\frac{\partial u_{p}^{\prime}}{\partial y}\right)\right]+\frac{\partial u_{p}^{\prime}}{\partial y} \frac{\partial}{\partial y}\left[\frac{\partial}{\partial y}\left(\frac{\partial v_{p}^{\prime}}{\partial x}\right)\right]\right\} \\
& =\frac{\partial}{\partial y}\left[\varepsilon_{p} \frac{\mu_{t p}}{S c}\left(\frac{\partial u_{p}}{\partial y}\right)\right]
\end{aligned}
$$

The seventh expression in the right-hand side of Eqn. (34) can be developed

$$
2 \mu_{p}\left(\frac{1}{\rho_{p}} \frac{\partial u_{p}^{\prime}}{\partial y} \frac{\partial F_{x}^{\prime}}{\partial y}+\frac{1}{\rho_{p}} \frac{\partial v_{p}^{\prime}}{\partial x} \frac{\partial F_{y}^{\prime}}{\partial x}\right)=\phi_{\varepsilon}
$$

Substituting Eqns. (35) $\div(42)$ into Eqn. (34), we get the following equation:

$$
\begin{aligned}
& \rho_{p} \frac{\partial \varepsilon_{p}}{\partial t}+\rho_{p} u_{p} \frac{\partial \varepsilon_{p}}{\partial x}+\rho_{p} v_{p} \frac{\partial \varepsilon_{p}}{\partial y} \\
& =\frac{\partial}{\partial y}\left[\frac{\mu_{p}}{\sigma_{\varepsilon}}\left(\frac{\partial \varepsilon_{p}}{\partial y}\right)\right]+\frac{\partial}{\partial y}\left[\varepsilon_{p} \frac{\mu_{p}}{S c}\left(\frac{\partial u_{p}}{\partial y}\right)\right]+\mu_{p} C_{\varepsilon 1} \frac{\varepsilon_{p}}{k_{p}}\left(\frac{\partial u_{p}}{\partial y}\right)^{2}+C_{\varepsilon 2} \frac{\varepsilon_{p}^{2}}{k_{p}}+\phi_{\varepsilon}
\end{aligned}
$$

The experimental coefficients should be (Schraiber 1987)

$C_{\mu}=0.09 ; C_{\varepsilon 1}=1.44 ; C_{\varepsilon 2}=1.92 ; \sigma_{k}=1 ; \sigma_{\varepsilon}=1.3$.

\section{Conclusions}

The four-equation turbulence model includes Eqns. (44) $\div(47)$.

$$
\rho_{g} \frac{\partial k_{g}}{\partial t}+\rho_{g} u_{g} \frac{\partial k_{g}}{\partial x}+\rho_{g} v_{g} \frac{\partial k_{g}}{\partial y}=\frac{\partial}{\partial y}\left[\frac{\mu_{g}}{\sigma_{k}}\left(\frac{\partial k_{g}}{\partial y}\right)\right]+\mu_{g}\left(\frac{\partial u_{g}}{\partial y}\right)^{2}-\rho_{g} \varepsilon_{g}-\varepsilon^{*}
$$




$$
\begin{aligned}
& \rho_{p} \frac{\partial k_{p}}{\partial t}+\rho_{p} u_{p} \frac{\partial k_{p}}{\partial x}+\rho_{p} v_{p} \frac{\partial k_{p}}{\partial y}=\frac{\partial}{\partial y}\left[\rho_{p} \frac{\mu_{p}}{\sigma_{k}}\left(\frac{\partial k_{p}}{\partial y}\right)\right]+\frac{\partial}{\partial y}\left[k_{p} \mu_{p}\left(\frac{\partial u_{p}}{\partial y}\right)\right]+\mu_{p}\left(\frac{\partial u_{p}}{\partial y}\right)^{2}-\rho_{p} \varepsilon_{p}+\varepsilon^{*} \\
& \rho_{g} \frac{\partial \varepsilon_{g}}{\partial t}+\rho_{g} u_{g} \frac{\partial \varepsilon_{g}}{\partial x}+\rho_{g} v_{g} \frac{\partial \varepsilon_{g}}{\partial y}=\frac{\partial}{\partial y}\left[\frac{\mu_{g}}{\sigma_{\varepsilon}}\left(\frac{\partial \varepsilon_{g}}{\partial y}\right)\right]+C_{\varepsilon 1} \mu_{g} \frac{\varepsilon_{g}}{k_{g}}\left(\frac{\partial v_{g}}{\partial y}\right)^{2}-C_{\varepsilon 2} \frac{\varepsilon_{g}^{2}}{k_{g}}-\phi_{\varepsilon} \\
& \rho_{p} \frac{\partial \varepsilon_{p}}{\partial t}+\rho_{p} u_{p} \frac{\partial \varepsilon_{p}}{\partial x}+\rho_{p} v_{p} \frac{\partial \varepsilon_{p}}{\partial y}=\frac{\partial}{\partial y}\left[\frac{\mu_{p}}{\sigma_{\varepsilon}}\left(\frac{\partial \varepsilon_{p}}{\partial y}\right)\right]+\frac{\partial}{\partial y}\left[\varepsilon_{p} \frac{\mu_{p}}{S c}\left(\frac{\partial u_{p}}{\partial y}\right)\right]+\mu_{p} C_{\varepsilon 1} \frac{\varepsilon_{p}}{k_{p}}\left(\frac{\partial u_{p}}{\partial y}\right)^{2}+C_{\varepsilon 2} \frac{\varepsilon_{p}^{2}}{k_{p}}+\phi_{\varepsilon}
\end{aligned}
$$

Application of the $k_{g}-k_{p}-\varepsilon_{8}-\varepsilon_{p}$ turbulent model with additional equations for the turbulent kinetic energy $k_{p}$ and the turbulent dissipation rate $\varepsilon_{p}$ of the second phase seems to be worthwhile for simulating two-phase non-homogeneous turbulent flows in industrial combustor.

\section{Nomenclature}

$\begin{array}{ll}u_{g} ; v_{g} & : \text { Velocity components of the gas phase } \\ u_{p} ; v_{p} & : \text { Velocity components of the second phase } \\ u_{g}{ }^{\prime} ; v_{g}{ }^{\prime} & : \text { Fluctuation of velocity components of the gas phase } \\ u_{p}{ }^{\prime} v_{p}{ }^{\prime} & : \text { Fluctuation of velocity components of the second phase } \\ \bar{u}_{g} ; \bar{v}_{g} & : \text { Mean of velocity components of the gas phase } \\ \bar{u}_{p} ; \bar{v}_{p} & : \text { Mean of velocity components of the second phase } \\ \rho_{g} ; \rho_{p} & : \text { Density of the gas phase and the second phase } \\ p & : \text { Pressure } \\ \varepsilon_{g} ; \varepsilon_{p} & : \text { Turbulent dissipation rate of the gas phase and the second phase } \\ k_{g} ; k_{p} & : \text { Turbulent kinetic energy of the gas phase and the second phase } \\ \mu_{g} ; \mu_{p} & : \text { Dynamic molecular viscosity of the gas phase and the second phase } \\ \tau_{g} ; \tau_{i j} & : \text { Specific Reynolds stress tensor of the gas phase and the second phase } \\ F_{x} ; F_{y}{ }^{\prime} & : \text { Forces } \\ C_{\varepsilon 1} ; C_{\varepsilon 2} & : \text { Dissipation rate coefficients. } \\ S_{i j} & : \text { Mean strain rate tensor. } \\ \sigma_{k} & : \text { Turbulent Prandtl for kinetic energy } \\ \sigma_{\varepsilon} & : \text { Turbulent Prandtl for dissipation rate } \\ f_{\varepsilon 1} ; f_{\varepsilon 2} & : \text { Boundary functions } \\ \mu_{L} & : \text { Laminar viscosity } \\ \mu_{T} & : \text { Turbulent viscosity } \\ \varepsilon^{*} & : \text { Eddy viscosity } \\ \phi_{\varepsilon} & : \text { Explicit wall term }\end{array}$




\section{REFERENCES}

Blazek, J., Computational Fluid Dynamics: Principles and Applications. Elsevier, 2001.

Chou, P. Y., On Velocity Correlations and the Solutions of the Equations of Turbulent Fluctuations. Quart. of Appl. Math. 3, 1945.

Erlebacher, G., Hussaini, M. Y., Speziale, C. G., and Zang, T. A., Toward the Large-Eddy Simulation of Compressible Turbulent Flows, J. Fluid Mech. 238 (1992) 155-85.

Jones, W. P., Launder, B. E., The Prediction of Laminarization with a Two-Equation Model of Turbulence. Int. Journal of Heat and Mass Transfer, 15, 1972.

Jones, W. P., Launder, B. E., The Prediction of Low Reynolds Number Phenomena with a TwoEquation Model of Turbulence. Int. Journal of heat and mass transfer, 16, 1973.

Launder, B. E., Sharma, B. I., Application of the Energy Dissipation Model of Turbulence to the Calculation of Flow Near a Spinning Disc. Letters in Heat and Mass Transfer, 1, 1974.

Launder, B. E., Spalding, B., The Numerical Computation of Turbulent Flows, Comput. Methods Appl. Mech. Eng. 3, 1974.

Schraiber, A. A., Gas Turbulent Flows, (Naucova Dumca), 1987.

Spalart, P. and Allmaras, S., A One-Equation Turbulent Model for Aerodynamic Flows, AIAA Paper 92-0439, 1992.

Wilcox, D. C., Reassessment of the Scale Determining Equation for Advanced Turbulence Models. AIAA Journal 26, 1988.

\section{Engineering International !!!}

"Speedy publication service, Online archives, Paperless, web-based peer review system, Open access policy, Indexing in world known citation databases, Global circulation, Broad international readership and authorship, Online submission system, Minimum publication charge" 